Euskal ikerketen aldizkaria | Revue d'études basques |

Revista de estudios vascos | Basque studies review

$10 \mid 2005$

Numéro $X$

\title{
Etude de génétique des textes à partir des textes d'E. Etxamendi
}

Joana Pochelu

(2) OpenEdition

Journals

Édition électronique

URL : http://journals.openedition.org/lapurdum/199

DOI : 10.4000/lapurdum.199

ISSN : 1965-0655

Éditeur

IKER

Édition imprimée

Date de publication : 31 décembre 2005

Pagination : 331-336

ISBN : 2-86781-409-X

ISSN : 1273-3830

Référence électronique

Joana Pochelu, «Etude de génétique des textes à partir des textes d'E. Etxamendi », Lapurdum [En ligne], 10 | 2005, mis en ligne le 01 avril 2008, consulté le 21 septembre 2020. URL : http:// journals.openedition.org/lapurdum/199; DOI : https://doi.org/10.4000/lapurdum.199 


\section{A propos de mon DEA. \\ (Obtenu en juin 2004, avec mention Très Bien, à l'Université de Michel de Montaigne-Bordeaux 3, au sein du DIEB : Département Inter-universitaire d'Etudes Basques, sous la direction de Mme Aurélie Arcocha-Scarcia)}

Où on parle de génétique textuelle...

Joana Pochelu

C’est dans le cursus des Etudes basques à la faculté de Bayonne, dans le cadre du Séminaire sur la Théorie Littéraire (Aurélie Arcocha-Scarcia) du niveau Maîtrise, que j’abordais la génétique des textes pour la première fois. Domaine qui m’a d'emblée attiré.

Puis pour le DEA, avec l'intention d'étudier quelques manuscrits de la Bibliothèque Nationale de France (BNF) à Paris, j'ai suivi un séminaire d'Introduction à la génétique des textes, de Pierre Marc de Biasi et Irène Fenoglio, à l'université de Paris 7 (Jussieu). Ce qui m’a amenée à étudier les manuscrits de travail d'un écrivain contemporain d'expression basque : Eñaut Etxamendi.

Dans la méthode critique du domaine littéraire, la génétique textuelle est l'un des seuls mouvements novateurs des trente dernières années. Aujourd'hui en France, l'ensemble des équipes de l'ITEM (Institut des Textes et Manuscrits modernes) CNRS-ENs (UMR 8132), participe à l'élaboration d'une théorie de la genèse du texte.

Il est nécessaire d'appliquer cette nouvelle méthodologie critique au texte d'expression basque. Afin d'y remédier, l'une des possibilités serait de fonder un lieu d'archivage. Par exemple en France, la BNF et les centres d'archives littéraires ont mené une politique d'acquisition de manuscrits assez active ces dernières années. Créé en 1988 à l'initiative de chercheurs et de professionnels de l'édition, l'IMEC (Institut Mémoire de l'Edition Contemporaine) par exemple, rassemble, préserve et met des fonds d'archives à la disposition du public. Le fonctionnement est particulier: les auteurs ou ayants droit y déposent leurs archives, en restant propriétaires de leur bien, et avec la possibilité de le récupérer à tout moment. Après réception du fond, et dès qu'il est traité, catalogué et côté, il est ouvert aux chercheurs, alors qu'à la BNF il faut attendre une dizaine d'années avant de pouvoir les consulter.

Il est donc nécessaire de créer un pôle, une institution de ce genre au Pays Basque, ou d'ouvrir un nouveau département au sein d'une bibliothèque ou centre de recherche comme IKER, afin que les écrivains d'expression basque puissent y déposer leur manuscrits, brouillons et autres papiers, et donner aux chercheurs la possibilité de travailler dans le domaine de la génétique du texte. Des manuscrits sont certes conservés à la bibliothèque Azkue d'Euskaltzaindia, à la bibliothèque Koldo Mitxelena, dans les fonds des députations ou des fonds privés, ainsi qu'à la bibliothèque municipale de Bayonne et dans les fonds d'archives du Musée basque, mais, comme nous l'avons dit plus haut, il faudrait un endroit spécifique pour que les écrivains y déposent leur manuscrits de travail et les mettent à disposition des généticiens. 
Mais qu'est exactement la critique génétique ? Comme l'explique Pierre-Marc de Biasi $^{1}$, son principe est celui d'une attention aussi grande que possible au travail de l'écrivain, à ses gestes, ses émotions, ses incertitudes : ce qu'elle propose c'est de redécouvrir le texte de l'œuvre à travers la succession des esquisses et des rédactions qui lui ont donné naissance et qui l'ont conduit à sa forme définitive.

Son projet est donc d'interpréter les manuscrits littéraires dans le but d'élucider de l'intérieur le travail de l'écrivain, le processus de l'écriture et la genèse des œuvres, sans accorder un statut privilégié au texte final dans lequel on pourra, lorsqu'il existe, ne voir qu'un dérivé ultime des précédentes métamorphoses ou une entité extérieure à la sphère de l'avant texte.

C'est ce à quoi je me suis employée pour mon sujet de DEA, autour de quatre dossiers de genèse (dont une nouvelle inédite) d'Etxamendi ; ceci, après avoir néanmoins, dans une première partie, présenté en détail la théorie de la génétique textuelle.

Ecrivain et chanteur, Eñaut Etxamendi écrit dans son dialecte du Pays de Cize-Garazi, sur le monde paysan et sur son enfance passée à la montagne: Elurra zikindurik (La neige salie), Azken elurra (La dernière neige) (1975), Laminak, Urtzoak (Les palombes), Aurora (1985), Gilentegiko Gilen (Gilen de Gilentegi), Gipuzkoako kutxa, 1988. Les dossiers génétiques que j'ai étudiés sont : Eiherzaintsaren auhena (Les lamentations de la gardienne du moulin), Gudarien eguna (Le jour des combattants), Izaiak (Les sapins) et Orbaizetako neskatxa (La fillette d'Orbaizeta), nouvelle inédite. Après avoir transcrit chaque page manuscrite, j'ai ensuite procédé à l'inventaire et à la description des pièces, afin de pouvoir analyser leur genèse. En voici ici en exemple, l'explication du dossier génétique de Gudarien Eguna, avec en regard, les deux feuilles manuscrites qui composent ce dossier.

\section{1) Transcription linéarisée}

Afin de pouvoir comprendre les différents mouvements d'écriture et de lecture que l'écrivant a effectués, j'ai tout d'abord transcrit linéairement les deux feuillets de ce dossier. Voici la convention utilisée pou la transcription :

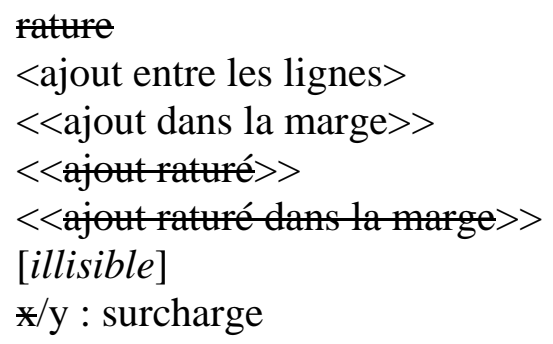

\section{Transcription du premier état}

Agur gudarien $<$ Argalara $>$ eguna

Hemen $<$ Euskadik ospa diona $>$ omendatzen duguna

Agur gudarien eguna

\footnotetext{
${ }^{1}$ Pierre Marc de Biasi, La génétique des textes, Nathan, Collection 128, 2000.
} 
Guzietan maitena

Oi zuen orhoitzapena

Izarkirik gindaukena

Izarkirik gindauken eta

Gaur zeruraino dohana

Bihotzian zauzkat gorderik

Agur bih/zueri bihotzetik

$\mathrm{Ni} /$ gutan zaude $<\mathrm{z}>$ tenak gorderik

Zuen irri-par ederretik

Malkoak egin zaizt begitik

Zuen orhoitzapenetik gatik

Ez nagoke sendatmikik

Ez nago-sendaturiketa

Herria da/jende $<<$ Jende $<$ Herri $>$ oro $>>$ odoldurik dolamenik $<$ dago zutik $>$

Herria $<<$ Jendea $<$ Herria $>$ ore $<$ өre $>>><$ dago zutik eta $>$ edoldurik etadolamenik eta

Zerua da odoldurik ere trixterik

$<<$ Itxaropenez beterik $>$

Ekia ekia emada/guk ekia

Hire suzko argi gorria <gemen $><<$ garbia argia $>>$

Gure gudarien [ezinir.]/auzia

Dagon munduan argia garbia

Orhoitzapenez bethia jauntzia

Eskualdun jende guzia

Eskual jende guzia eta

Zarete gure guardia

Gure guardia zaretela

Hil-hobitik begira

Eman diguzute odola

Zuen firma bezala

Orhoitzapena dohala

Arimake $<<$ oi gogoko $>>$ gordagila

Gogoko gordagila eta

Armak hartu ditzagula

\section{Transcription du second état}

\subsubsection{Gudarien eguna}

Agur Argalaren eguna

Euskadik ospa diona

Agur gudarien eguna

Egun orotan maitena

Oi ! Zuen orhoitzapena

Izarkirik gindaukena

Izarkirik gindauken eta 
Gaur zeruraino dohana

Agur zueri bihotzetik

Gutan zauztenak gorderik

Zuen irri-par ederretik

Malkoak zaizkit begitik

Zuen orhoitzapena gatik

Herri oro dago zutik

Herria dago zutik eta

Itxoipenez beroturik

Nai omen dutela bakea

Bainan hau dute xedea

Harmak lehenik guk uztea

Ta berenak beiratzea

Orhoitzapenen bidea

Zapalkuntzaz da betea

Zapalkuntzaz betea eta

Maltzurra (faltsua) dute elhea

Ortzia ! emaguk Ortzia

Lehengo gemen gorria

Gure gudarien auzia

Munduan dagon argia

Orhoitzapenez josia

Duzute Euskal jendia

Euskal jende guzia eta

Zarete gure guardia

Gure guardia zaretela

Izar-begiz begirala

Eman diguzute odola

Zuen izenpe bezala

Orhoitzapena dagola

Su-dirdiretan horrela

Dirdiretan horrela eta

Abarkak jauntz ditzagula.

\section{2) Inventaire et description des pièces}

Le présent dossier est composé des manuscrits de travail d'une chanson interprétée par Etxamendi et Larralde, parue dans le disque Antologia, (Donostia, Elkarlanean, 1998)).

\section{Feuillet 1}

Le premier feuillet est le premier état du manuscrit autographe du poème, il s'agit d'un brouillon. Une partition autographe, mélodie de la chanson, précède le texte. 


\section{Feuillet 2}

Le second et dernier feuillet de ce dossier est la mise au net de l'état antérieur. Il correspond au manuscrit définitif : c'est le dernier état autographe de l'avant-texte, qui donne l'image du modèle sur lequel sera reproduite la version imprimée.

\section{3) Genèse du poème Gudarien eguna}

\section{Etat 1}

Nous avons donc ici le premier état du poème, précédé par une partition ; ce qui pourrait laisser supposer que la musique à été écrite avant les paroles.

Il semblerait que l'opération scripturale de ce premier jet ne contienne qu'une seule campagne d'écriture. En effet, le texte montre un tracé continu, les trois premières strophes forment une colonne diagonale, et la quatrième strophe, écrite en vis-à-vis de la première, suit la même diagonale. De plus, si la graphie du début est appliquée, nous pouvons constater qu'au fur et à mesure de l'écriture, la main du scripteur fait diligence, les lettres, les mots ont été tracés à toute allure, ce qui explique l'inclinaison du texte. L'empressement de l'écrivant semble refléter la hâte qu'il a de voir arriver le jour des combattants.

Puis, à la suite du premier jet, l'auteur a procédé à deux campagnes de corrections, liées bien évidemment à deux campagnes de relecture. Dès la première relecture, il a procédé à une campagne de corrections, en raturant spécialement la deuxième strophe. Lorsqu'il rature, il fait systématiquement des ajouts interlinéaires ou marginaux. A une exception près : le premier vers de la deuxième strophe est raturé, et le segment substitutif est placé au dessous du segment barré, toutefois il n'est pas interlinéaire, mais se trouve en lieu et place d'un vers. Nous pouvons conclure que la correction est faite au fil de l'écriture. Chose que nous pouvons également observer sur les vers sept et neuf de la même strophe.

Lors de la seconde campagne de relecture l'écrivant a corrigé les corrections qu'il avait faites dans la marge : il rature le segment substitutif qui sera substitué à son tour (vers 9 et 10)

D’autre part, il est décidé dès le début, que le septième vers de chaque strophe sera la répétition du sixième, ce qui sera maintenu jusqu’à la forme définitive. Ce phénomène de répétition est pensé au fil même de la plume, puisque l'écrivant a souligné la conjonction de coordination « eta » (et) en fin de vers sept de toutes les strophes, comme pour se le rappeler à lui-même ainsi que pour se le confirmer. Le huitième vers de la deuxième strophe amène la garantie que le soulignement a bien été effectué dans le courant de l'écriture car en effet, « eta » est souligné, et comme nous l'avons dit plus haut, ce segment à été raturé durant l’écriture même.

« Orhoitzapena » (le souvenir) est invariablement évoqué dans le vers précédant ces répétitions. Ce fait est signalé par un soulignement du vers entier. Dans la troisième strophe par contre, il a souligné le vers d'en dessous, sans doute par mégarde.

Ainsi, sur le brouillon l’auteur insiste donc sur les répétitions de manière significative.

\section{Etat 2}


Sur ce feuillet, contrairement au premier jet, le titre est appliqué : Gudarien eguna (Le jour des combattants), et une date est apposée en haut de page, à gauche. Ainsi, nous apprenons que le poème a été écrit le 19 décembre 1982. La partition aussi est mise au net, et est daté du 5 décembre. Il s’agit bien de la date de création et non de mise au net, comme nous le prouve l'espace graphique : c'est d'abord le poème qui a été mis au propre et ensuite la partition. Les dates étant écrites dans cet ordre et non dans le sens inverse, alors oui, nous pouvons affirmer en toute logique que ce sont là les dates de production. Donc la supposition faite plus haut se voie confirmée : la musique a bien été écrite avant les paroles, et chacune, en une fois.

En plus de la mise au propre, des corrections immédiates ont été apportées. En outre, chose étonnante, une strophe a été ajoutée entre la deuxième et la troisième. Elle apparaît sans corrections, donc écrite en un seul jet, même si l'on y trouve une alternative non résolue. Ainsi, l'auteur ne sait pas si dans le texte imprimé il gardera «maltzurra » (rusé) ou « faltsua » (hypocrite), et met donc ce dernier entre parenthèses.

Des changements significatifs ont été apportés depuis le premier état : l'écrivain a transformé «ekia » en "ortzia »; il demande au souvenir de rester plutôt que de partir (strophe 5) ; enfin, il préfère chausser les abarka au lieu de prendre les armes (dernière strophe).

Il paraît évident dans ces brouillons, que l'écriture est le trésor de tous les possibles, que les suppressions, les changements donnent accès à ce qui aurait pu devenir texte, car durant le processus créatif, le texte a encore l'occasion de prendre diverses déviations. Il était au début beaucoup plus dur, plus explicite, mais finalement, Eñaut Etxamendi a décidé qu'il allait peser ses mots, que son chant des combattants allait être un peu plus tacite. Sur le texte imprimé, le poème a par ailleurs encore subi des changements.

Que dire de ce que l'on apprend de ce dossier ainsi que des trois autres étudiés ? Par exemple, et de manière très concise, lorsqu'il écrit des poèmes, Etxamendi couche sur le papier un premier jet auquel il apporte des changements après une relecture. D'autre part, nous avons remarqué également que lorsqu'il y a une partition sur le manuscrit, celle-ci est écrite avant le texte.

Mais pour pouvoir affirmer que cette procédure d'écriture est systématique chez cet auteur, il faudrait analyser d'autres de ses brouillons.

La critique génétique, jeune méthode critique des textes, pourrait être à même de prendre son essor au sein de la recherche sur la littérature d'expression basque. En juillet dernier, à l'initiative de l'ETEG (Euskal Testu Eta Genesiak) sous la direction d'Aurélie Arcocha-Scracia, équipe d'IKER (UMR 5478), a collaboré aux cours de l'Université d'été de Saint-Sébastien organisé par l'Université du Pays Basque (Euskal Herriko Unibertsitatea, Communauté Autonome Basque, Espagne), dédiés à la Génétique des textes, ou le processus créatif à travers les brouillons. En tant qu'écrivain, Bernardo Atxaga y a fait une communication en expliquant la genèse de quelques-uns de ses poèmes. D'autre part, l'édition Pamiela, pour faire la promotion de Soinujolearen semea (Le fils de l'accordéoniste, dernière publication d'Atxaga) a diffusé un brouillon de plan du village de Obaba, village où se déroule la narration. Seraient-ce là les prémices d’un intérêt pour la genèse des textes?

Si les écrivains allaient de plus en plus dans ce sens-là, les étudiants et chercheurs ne seraient-ils pas plus nombreux à s’intéresser à la génétique des textes ? Et qui sait ce que 
pourraient révéler les brouillons sur les processus de création dans la littérature contemporaine d'expression basque ?... 